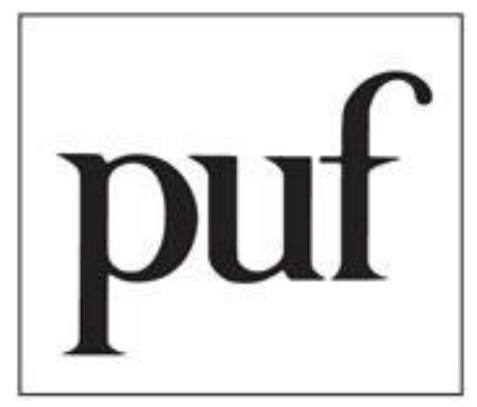

\title{
SUR LA RAISON DE LA GUERRE [KRIEGSGRUND]
}

Author(s): Petar Bojanic and Igor Krtolica

Source: Les Études philosophiques, No. 2, Nouvelles lectures de Rosenzweig (Avril 2009), pp. 195-205

Published by: Presses Universitaires de France

Stable URL: https://www.jstor.org/stable/20849961

Accessed: 23-10-2018 10:43 UTC

JSTOR is a not-for-profit service that helps scholars, researchers, and students discover, use, and build upon a wide range of content in a trusted digital archive. We use information technology and tools to increase productivity and facilitate new forms of scholarship. For more information about JSTOR, please contact support@jstor.org.

Your use of the JSTOR archive indicates your acceptance of the Terms \& Conditions of Use, available at https://about.jstor.org/terms

Presses Universitaires de France is collaborating with JSTOR to digitize, preserve and extend access to Les Etudes philosophiques 


\section{SUR LA RAISON DE LA GUERRE [KRIEGSGRUND]}

Und da bin ich nun (obne sagen zu können j'y suis j'y reste) $)^{1}$.

J'y suis, j'y reste

Le secret de cette expression qu'on attribue à Mac-Mahon tient à plusieurs choses. D'abord, d'après la légende, ce général ne se souvient pas avoir jamais prononcé ces mots; et, s'il les a prononcés, c'est après un fait d'armes : la conquête d'une " position » clé (la tour de Malakoff); enfin, s'il les a jamais prononcés, c'était en réponse à l'avertissement d'un général anglais lui affirmant la nécessité de se retirer, parce que sa position était malgré tout faible et qu'on pouvait prévoir une explosion catastrophique ('ennemi russe avait miné la tour au moment de se retirer). Bref, si ces mots courageux ont été prononcés, ils n'ont fait que manifester la résolution du général de ne pas faire machine arrière, quitte à succomber. En 1918 à Belgrade, et contrairement à ce général de la guerre de Crimée au sujet de laquelle il vient d'écrire, Franz Rosenzweig affirme qu'il ne peut pas répéter l'effort de Mac-Mahon ${ }^{2}$. Je suis là maintenant, à Belgrade, écrit Rosenzweig à sa mère, mais je ne peux pourtant pas dire: "J'y suis, j'y reste. " Je suis là, mais je ne peux pas y rester, je n'accepterai pas de dire « j’y suis, j'y reste ».

Je me permets ici de mettre entièrement au second plan la future capitulation de l'Allemagne, la paix quasiment assurée (ce sont les premiers mots

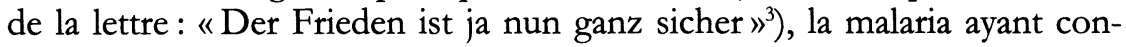
traint Rosenzweig à passer un mois dans un hôpital militaire de Belgrade, ses problèmes pour envoyer des lettres à sa mère à Cassel ou son attente quoti-

1. F. Rosenzweig, lettre du 13 octobre 1918, Der Mensch und sein Werk, in Gesammelte Schriften, 1. Briefe und Tagebücher, t. 2 : 1918-1929, La Haye, Martinus Nijhoff, 1979, p. 613.

2. Rosenzweig écrit sur la guerre de Crimée une année avant cette lettre dans le texte "L'Gcoumène ». Der Mensch und sein Werk, in Gesammelte Schriften, 3. Zweistromland. Kleinere Scbriften zu Glauben und Denken, La Haye, Martinus Nijhoff, 1982, p. 333 ; F. Rosenzweig, Confluences. Politique, histoire, judaïsme, trad. G. Bensussan, M. Crépon, M. de Launay, Paris, Vrin, 2003, p. 61.

3. Lettre du 13 octobre 1918, Briefe und Tagebücher, t. 2, p. 612.

Les Études philosophiques, n² 2/2009, p. 195-205 
dienne des nouvelles du train devant emmener les derniers blessés de l'Empire hors d'une Serbie honnie et jugée barbare'. La hâte de Rosenzweig - il est aujourd'hui facile de montrer qu'elle était injustifiée et que la malaria qu'il a contractée au sud de la ville de Niš l'a sauvé d'une mort certaine ${ }^{2}-$, sa défaite en tant que soldat et intellectuel allemand, restent dans l'ombre d'une défaite bien plus sérieuse que Rosenzweig admet définitivement à Belgrade. Dans les lettres du 13 et du 19 octobre adressées à sa mère, Rosenzweig affirme que la paix a eu pour conséquence que son opéra de guerre non publié [ungedruckte Kriegsopera] ou das blonde Putzanium, n'est définitivement plus d'actualité. Il est intéressant de remarquer que Rosenzweig ajoute immédiatement deux constats au fait que la guerre a pris fin et que sa " position ", inlassablement "conquise» tout au long de 1917, s'est écroulée: d'une part, il constate la ruine de l'idée d'une Europe centrale (Mitteleuropa ist Essig) et la futilité de la théorie de Friedrich Naumann, fer de lance de la pensée centreuropéenne ${ }^{3}$; d'autre part, il constate avec surprise que luimême, durant toute la guerre, souhaitait un roi et était monarchiste ${ }^{4}$ !

Rosenzweig suppose que cette position est tellement intenable qu'elle ne lui permet absolument plus de rester là où il est, de la défendre et, dès lors, de dire : «J'y suis, j'y reste.»

Que se passe-t-il donc en 1917 et en quoi consiste le projet impossible et avorté de Rosenzweig ? Pour préserver, voire pour corriger ses intentions, ne doit-on pas le penser avec Rosenzweig, mais contre Rosenzweig luimême ? Est-il de toute manière possible de reconstruire cette tentative et de poursuivre ce livre resté inachevé ? Ou bien doit-on, seulement maintenant, commencer à écrire ce livre sur la guerre (ein Buch über den Krieg)?

Il existe d'autres séries de questions qui introduiraient une incertitude encore plus grande à l'égard du statut du projet rosenzweigien. L'enquête pourrait porter sur la supposition qu'un tel projet dépend strictement du fait

1. Dans le texte "Deutschland und der Weltkrieg" (29 août 1914), Friedrich Meinecke dit que l'Autriche et l'Allemagne ont été provoquées à entrer en guerre car l'État et le peuple serbes étaient incapables de mener une guerre loyale [loyale Krieg] et honnête, et menaient déjà une politique fanatique, barbare et criminelle. Meinecke insiste sur le fait qu'il comprend parfaitement le désir de la Serbie de fonder son propre État national, mais, pour qu'un peuple puisse y parvenir, il est nécessaire qu'il montre d'abord qu'il est un peuple de culture. [Kulturvolk]. F. Meinecke, Politische Schriften und Reden, in Werke, t. 2, Darmstadt, Siegfried Töche-Mittler Verlag, 1958, p. 96-97.

2. Je pense ici aux crimes commis contre les armées défaites d'Autriche et d'Allemagne, survenus sur le territoire de Serbie au cours des derniers mois de la guerre.

3. "Les Centreuropéens ne peuvent pas créer l'Europe centrale, mais c'est l'Europe centrale qui peut créer les Centreuropéens » (Briefe und Tagebücher, t. 2, p. 612). Friedrich Naumann est certainement la première référence des réflexions géopolitiques de Rosenzweig du tout début de la guerre, et ce jusqu'aux dernières années de Rosenzweig. Rosenzweig lit les articles de journaux de Naumann pendant la guerre et se réfère souvent aux idées (allemandes) d'une « Nouvelle orientation », d'un « objectif de guerre », d'une « Europe centrale », de «la guerre nécessaire comme créatrice d'un esprit d'Europe centrale », comme dans le premier chapitre programmatique du livre de Naumann Mitteleuropa, «Der gemeinsame Krieg und seine Folgen " (Berlin, G. Reimer Verlag, 1915, p. 1-32).

4. Lettre du 19 octobre 1918, F. R., Briefe und Tagebücher, t. 2, p. 614. 
que la guerre dure. Il semble que la paix est l'ennemi déclaré de l'écriture sur la théorie des causes et des buts de la guerre. Dans ce cas, il serait important de prêter attention aux jeux patriotiques de Rosenzweig, à l'influence de la propagande de guerre sur ses textes ${ }^{1}$, sur ses analyses des nouvelles journalistiques et à sa tentative permanente d'anticiper les mouvements sur le front et dans la politique mondiale, c'est-à-dire de caler la construction de son texte sur le rythme de la guerre. Comment comprendre son désespoir et sa totale perte de foi dans l'issue de la guerre (c'est-à-dire dans le destin de son texte) quand il apprend la destitution du Reichskanqler Bethmann-Hollweg, patriote modéré qui était, selon Rosenzweig, conscient de la signification de la guerre pour la formation d'un nouvel ordre mondial ${ }^{2}$ ? Comment comprendre ses nombreux commentaires brutaux et hâtifs, sa rage à l'égard de l' " issue de la guerre » (Kriegsausgang) ( "Es [le peuple anglais] ist ein barbarisches Volk. Il est vraiment malheureux que ce soient eux [les Anglais] qui aient gagné la guerre ») $)^{3}$ ? Ou bien encore, comment comprendre qu'il ait attendu avec autant d'impatience de savoir si l'Amérique et le Japon allaient ou non entrer en guerre et qu'il ait brusquement changé de paradigme dans la construction de son texte (le texte "Thalatta. Hégémonie maritime et liberté des mers ", de plus de 40 pages dactylographiées, a été écrit du 23 au 27 décembre 1917)?

La manière dont a écrit Rosenzweig, et la vitesse à laquelle il l'a fait, nous conduisent à une problématique encore plus complexe, quant au statut du texte qui a été écrit (et n'a pas été détruit) et dont la publication a été repoussée. Je ne pense pas ici seulement à l'inintelligibilité définitive des " politiques de l'archive », essentiellement inacceptables et toujours obscures (der Dunkelheit der Archive) $)^{4}$, mais à quelques problèmes techniques précis dans la reconstruction des manuscrits, de même qu'à ce que j'appellerais les « fictions des archives». Il est impossible de reconstruire correctement les textes écrits sur des cartes postales et sur de petits papiers cartonnés, de même qu'il est impossible d'imprimer dans les textes publiés les commen-

1. Cf. M. Crépon et M. de Launay, «Introduction» à F. Rosenzweig, Confluences. Politique, bistoire, judaïsme, 2003, p. 15.

2. Lettre du 20 juillet 1917, 1. Briefe und Tagebücher, t. 1, p. 422-423. Cf. P. Mendes-Flohr, Divided Passions. Jewish Intellectuals and the Experience of Modernity, Detroit, Wayne State University Press, 1991, p. 323-325.

3. C'est un commentaire de Rosenzweig écrit à l'occasion de la visite qu'il a rendue à ses cousins [die Londoner], qu'il n'avait pas vus depuis neuf ans et chez qui il reconnait un léger "changement d'essence " [Englischkeit des Wesens]. Lettre du 6 juillet 1920. F. Rosenzweig, Die Gritli-Briefe: Briefe an Margrit Rosenstock-Hussey, Tübingen, Bilam Verlag, 2002, p. 621.

4. Kant parle de "l'obscurité des archives» dans la Métaphysique des mours, au \61. Quelques fragments très intéressants du «Journal» de Rosenzweig sont, sans nécessité aucune, restés non publiés. Pourtant, le document le plus significatif, resté non publié mais qui est décisif pour la compréhension des textes " de guerre » de Rosenzweig, est la liste des livres qu'il a lus de février 1916 à août 1918 (Bücherliste). Ce document porte le numéro "V » et fait 7 pages. Franz Rosenzweig Collection; AR 3001 ; box 1 ; folder 3 ; Leo Baeck Institute at the Center for Jewish History. Les livres sont mentionnés selon le mois où Rosenzweig les a lus, et il a au total noté plus de 300 titres. 
taires marginaux que Rosenzweig a notés après coup ${ }^{1}$. Mis à part cela, il est pratiquement impossible de faire une édition critique des " textes de guerre » de Rosenzweig, car ils s'inspirent souvent de textes et de commentaires journalistiques qu'il n'a jamais cités. Finalement, tous ces textes qui n'ont jamais été imprimés et ne sont jamais devenus publics réveillent sans cesse des fantasmes quant à leur «influence fictive» sur d'autres auteurs de la même époque et sur leurs propres textes.

Son projet de guerre ${ }^{2}$ - nous le nommons " projet de guerre » et admettons qu'il n'a jamais été qu'un "projet» génial -, Rosenzweig lui donne d'autres noms. Hormis Kriegsopera et Putrianum (l'inspiration provient de son cousin Victor Ehrenberg surnommé Putzi, et avec lequel Rosenzweig élabore des projets communs), il l'appelle également Hansiaca (car il fait un projet similaire sur un livre en commun avec son ami Hans Ehrenberg), puis Kriegsausgang et Kriegsgrund, ou encore Theatrum Europaeum. Ein Versuch über den Schauplatz der Weltgeschicbte ${ }^{3}$. Dans trois lettres envoyées à trois adresses différentes au cours du premier semestre de $1917^{4}$, Rosenzweig explique en détail l'origine de son idée d'un grand livre sur la guerre et annonce finalement qu'il en a commencé la rédaction, conscient qu'il ne pourra sûrement pas faire sur le front tout ce qu'il escompte. Il nous semble nécessaire d'admettre que le texte Globus - le plus long parmi la dizaine d'autres textes - constitue la partie fondamentale de ce premier livre imaginé et que les autres textes en sont des ajouts et des parties miniatures. Rosenzweig déclare qu'en 1910 ou en 1911, c'est-à-dire durant la rédaction de sa thèse sur Hegel et l'État, il avait l'intention d'écrire une histoire de la raison de la guerre (Kriegsgrund). Il s'empresse d'achever au plus tôt son doctorat, pour se consacrer au plus vite à ce travail, car le 25 novembre 1910 naît la Fondation Carnegie qui doit précisément financer des projets sur le problème de l'ori-

1. Un parfait exemple en est l'un des textes les plus importants de Rosenzweig sur la guerre, écrit en décembre 1917 : «Cannes et Gorlice. Une explication du concept stratégique d'espace. " Dans le manuscrit, Rosenzweig a par exemple systématiquement effacé le mot Vermichtungsstrategie et l'a remplacé par le mot absolute Krieg. Cette substitution est inhabituelle de même que le long fragment consacré à Clausewitz qui se trouve à la deuxième page du manuscrit (Confluences. Politique, bistoire, judaïsme, p. 126; GS, Zweistromland, p. 284). Franz Rosenzweig Collection; AR 3001 ; box 2 ; folder 34 ; Leo Baeck Institute at the Center for Jewish History.

2. Onze textes de Rosenzweig de 1917 qui "contiennent» ce projet n'ont pas été publiées sous le nom de leur auteur (quatre ont été publiées sous un pseudonyme). Dans les Gesammelte Schriften, ces textes ont été classés sous le titre "Zur Politik», tandis qu'au Leo Baeck Institute ces textes divisés en trois groupes : 1. "Globus », 2. "Vox Dei ? » et "Cannä und Gorlice » sont dans un grand groupe de textes qui porte le titre "Other », tandis que les huit autres textes se trouvent insérés sous le titre 3. "Prolegomena zur Politik».

3. Francesko Paolo Ciglia, le directeur de l'édition et de la traduction italienne du texte Globus. Per una teoria storico-universale dello spazio (trad. S. Carretti, Genova, Marietti 1820, 2007), a ajouté à l'étude de Rosenzweig un repérage détaillé des lettres et notes qui permettent de reconstruire son projet. Cf. «Sezione dokumentaria. Lettere e appunti personali sul Globus », p. $113-140$.

4. Il s'agit des lettres aux parents du 11 janvier 1917, à Rudolf Ehrenberg du 29 mars 1917 et à Gertrud Oppenheim du $1^{\text {er }}$ mai 1917 (F. R., Briefe und Tagebücher, t. 1, p. 334-335, 375, 395). 
gine et des causes de la guerre 1 . Dans ces trois fragments de janvier, mars et mai 1917, Rosenzweig précise encore quelques éléments : qu'il souhaite analyser les guerres depuis 1494 jusqu'à aujourd'hui ; qu'il est particulièrement intéressé par le rapport entre la raison de la guerre (Kriegsgrund) et les débuts de la guerre (Kriegsanfängen); qu'il écrit en ce moment prioritairement au sujet de l'actualité (des aktuellen Ausgangs); qu'il avait eu l'intention de travailler aux archives diplomatiques et d'examiner tous les facteurs de la guerre, c'est-àdire les raisons qui font que les guerres commencent; qu'il est absolument incapable d'écrire un tel livre aujourd'hui ; que, s'il écrivait ce livre aujourd'hui, il ferait partie d'un livre plus grand (als Teil eines grösseren), qui demande encore du temps ; que c'est pour cette raison qu'il doit à nouveau " avorter " le projet de ce livre...

Il semble aujourd'hui impossible de reconstruire précisément le « début » de ce projet qu'évoque Rosenzweig. Il n'existe aucune note datant de la période de rédaction de son doctorat pouvant nous confirmer que Rosenzweig avait bien l'intention d'écrire un livre sur la raison de la guerre, de même qu'il n'existe aucune trace de la Kriegsgrund dans son doctorat publié (l'avant-propos de sa thèse publiée Hegel et l'État a été écrit après la guerre, en mai 1920 à $\mathrm{Cassel}^{2}$ ). Quelques mois après sa soutenance de thèse, Rosenzweig parle de manière très inspirée de la nouvelle guerre, de la guerre mondiale qui approche, qui est en vue, et dont nous ne connaissons ni la forme ni la morale :

Nous ne sommes pas à l'orée d'une guerrè [einem Krieg], mais d'une époque de guerres [Epoche von Kriegen], et du point de vue de l'Europe nous sommes déjà à l'intérieur d'une telle époque [und europäisch gesebn sind wir sogar schon drin] ${ }^{3}$.

C'est tout. Cependant, il n'y a aucune raison pour exclure la possibilité que Rosenzweig ait pu imaginer ce projet comme un ajout parfait à la théorie hégélienne de la guerre. De même, en d'autres endroits de sa correspondance, Rosenzweig place son projet sur le même plan que son doctorat (que son «Hegel »). D’ailleurs, Rosenzweig compare à plusieurs reprises sa position dans la guerre avec celle de Hegel à Iéna. Toutefois, il est peutêtre nécessaire de faire tout de suite quelques réserves à l'égard des autointerprétations de Rosenzweig quant à l' «identité" de registre entre ses deux conceptions radicalement différentes. En effet, dans son doctorat, et probablement comme personne auparavant, Rosenzweig a déconstruit (et démoli, en même temps) quelques points clés de la théorie hégélienne du droit soutenant précisément la pensée de la guerre et celle de l'État, qui

1. La Fondation Carnegie était à l'époque organisée en trois sections : la deuxième avait pour objectif « to study the causes and impact of war».

2. F. Rosenzweig, Hegel et l'État, trad. G. Bensussan, Paris, PUF, 1991, p. 3-10; Hegel und der Staat, Aalen, Scientia Verlag, 1962 (1920), t. 1, p. V-XIII.

3. Lettre à Hans Ehrenberg, fin novembre 1912, 1. Briefe und Tagebücher, t. 1, p. 124.

4. Dans la lettre à Mawrik Kahn, qu'il a vraisemblablement écrite à l'automne 1919, Rosenzweig qualifie son texte "Globus » et son "Hegel» de " manuscrits morts " [gestorbene Schriften] (Briefe und Tagebücher, t. 2, p. 652). 
« repose sur les armes »(Staat aus Waffen gestellt) $)^{1}$ le concept de violence et de légitimation par la violence, le sacrifice et le sacrifice pour la patrie, le lien entre patriotisme et souveraineté, l'analogie de la souveraineté et de l'organisme, etc. Il est intéressant de constater que Rosenzweig ne revient sur ces thèmes que dans un texte de 1917, à l'occasion d'une analyse du sacrifice pour la patrie, mais dans un contexte relativement différent et sans la moindre verve antihégélienne ${ }^{2}$. Excepté cela, le projet de Rosenzweig sur la guerre n'est-il pas conçu pour effectuer précisément ce que Hegel n'a pas effectué - pour penser la guerre, pour trouver et donner un sens à la guerre, non pas à la guerre comme élément constitutif d'un État (ou de quelque État que ce soit, voire d'un seul État mondial), mais comme facteur clé de la création d'un monde sans États, d'un monde sans frontières, et de la paix ${ }^{3}$ ? Dans cette optique, ce projet est dès son origine et dans son intention même un projet absolument hégélien, bien que son actualisation, c'est-à-dire la recherche concrète par Rosenzweig des raisons et des buts de la guerre, aboutisse à des résultats radicalement antihégéliens et l'éloigne définitivement de son mentor Meinecke.

"Ainsi, cette guerre n'a pas été, comme l'affirme Meinecke, politiquement improductive ainsi qu'inutile [zwecklos]. (...) L'erreur fondamentale [Grundfebler] de Meinecke est qu'il continue malgré tout à penser aux États [Staaten], et non pas à des fédérations d'États [Staatenverbänden]. Il dit: les fédérations d'États font que les guerres sont inutiles, que les guerres n'apportent rien de politiquement créatif, et c'est pourquoi Meinecke veut dire que les guerres participent de manière créative exclusivement aux États individuels. Mais les États ne sont plus du tout les porteurs de l'histoire, car ce sont les fédérations d'États, et c'est précisément sur eux qu'agit de manière créative la guerre, et avant tout cette guerre. Le noyau de l'idée pacifiste, fondé sur les exigences de la Realpolitik [realpolitisch berecbtigte Kerm], est : le dépassement du national dans un État fédéral [die Überwindung des Nationalen im Verbandsstaat]. $»^{4}$

Cet extrait d'une lettre à ses parents fait partie d'une réaction très vive et très nerveuse de Rosenzweig à l'engagement pacifiste de Meinecke et à son texte de septembre 1917 (Demobilmacbung der Geister) $)^{5}$. Ce n'était pas seule-

1. F. Rosenzweig, Hegel et l'État, p. 134 ; Hegel und der Staat, t. 1, p. 133.

2. Cf. "Vox Dei ? Le cas de conscience de la démocratie », F. R., Confluences. Politique, bistoire, judaïsme, p. 103-119; GS, Zweistromland, p. 267-282.

3. « (...) l'État en guerre prend dans ce conflit une forme qui le conduit hors de ses frontières en temps de paix, aussi bien extérieurement qu'intérieurement ; l'État en guerre est l'image future d'un État à venir lorsque la paix sera rétablie ». Cf. "Cannä und Gorlice », F. R., Confluences. Politique, histoire, judaïsme, p. 138 ; GS, Zweistromland, p. 294.

4. Lettre du $1^{\text {er }}$ octobre 1917, 1. Briefe und Tagebücher, t. 1, p. 459. Les commentaires sur Meinecke se poursuivent aussi dans les lettres suivantes de cette période.

5. Dans sa correspondance, Rosenzweig ne mentionne nulle part le titre de ce texte qui a été publié dans le Frankfurter Zeitung und Handelsblatt du 23 septembre 1917 (le texte a été à nouveau imprimé dans F. Meinecke, Politische Schriften und Reden, in Werke, t. 2, p. 195-200). Meinecke est la deuxième référence principale des textes géopolitiques de Rosenzweig. Dans la lettre à ses parents du 30 janvier 1917 (p. 342), Rosenzweig note, hormis Naumann et Meinecke, les noms de Kjellen, Leusch, Tröltsch, Simmel, Ranke, comme ceux qui influencent 
ment pour Rosenzweig une bonne occasion d'exprimer à nouveau ses réserves à l'endroit du pacifisme, de montrer en détail ses limites et son origine militaire (ainsi que profane), ou bien d'accentuer son incrédulité à l'égard du conservatisme de son maitre, mais également d'expliquer qu'il n'y a pas de paix mondiale, même si les États et les esprits (les intellectuels) deviennent pacifistes et se "démobilisent».

Comme si pour Rosenzweig la guerre ne devait pas se terminer avant d'avoir accompli son rôle fondamental : produire l'espace mondial commun et l'unité des peuples, supprimer les États, déplacer et transformer les frontières ${ }^{1}$. Comme si ce court texte programmatique de Friedrich Meinecke forçait, mais aussi anticipait, la fin à venir de l'inimitié entre les États et la fin de la Première Guerre mondiale. Ce qui est sûr, c'est qu'il est désormais possible de comparer l'énervement de Rosenzweig après la lecture de ce pamphlet avec les deux épilogues de son texte Globus. Je pense d'abord au dernier chapitre de la première partie ( "L'Ecoumène») qui s'intitule "Monde» et dans lequel Rosenzweig affirme que «les plus grandes luttes, les combats pour la véritable idée du monde sont encore à venir » - et dans la dernière phrase, il conclut, en somme, que le monde est a priori un, car Dieu, « le guerrier » (Kriegsmann), l'a ainsi créé ${ }^{2}$; je pense également à la fin de la deuxième partie ( "Thalatta»): il me semble que Rosenzweig a écrit et l'un et l'autre passage pendant ces trois jours-là, à la fin de 1917.

Ainsi, à la fin de «Thalatta » - la fin de ce texte est en même temps la fin du texte Globus, la fin de la tentative de Rosenzweig, la fin et l'interruption du projet de guerre, la trace ou le résidu du « livre avorté » qui n’a jamais été écrit, Rosenzweig affirme que les frontières et les divisions mondiales ont

de manière décisive l'exécution de son projet sur la guerre (le texte de Ranke de 1833, «Die grossen Mächte ", est particulièrement significatif aux yeux de F. R. ; F. Meinecke a arrangé un tiré-à-part du texte de Ranke et l'a publié en 1916). À ces auteurs, j'ajouterais les titres de quelques livres que Franz Rosenzweig a lus et sans lesquels sa tentative n'aurait pas été possible : au mois de juillet 1916, F. R. lit le petit livre de Julius Kierst, Die antike Idee der CEkumene in ibrer politischen und kulturellen Bedeutung (1903); en janvier 1917, le livre d'Eugen Schiffer, Vom Kriegsgrund zum Kriegsziel (1889); en mai 1917, celui de Clausewitz, Vom Kriege (18161830). Franz Rosenzweig Collection; AR 3001 ; box 1 ; folder 3 ; Leo Baeck Institute at the Center for Jewish History.

1. Dans l'introduction du texte "Globus », qu'il écrit quand l'ensemble du projet est achevé, Rosenzweig dit : «Être délimitée [Begrenzbarkeit] par des frontières est sa nature [à la terre], l'absence de toute frontière [Unbegrenztheit] n'est que sa fin dernière. » Au début de "L'CEcoumène", Rosenzweig poursuit : "Si ce ne sont les forces en présence, leurs fins du moins justifient certainement le qualificatif de la guerre actuelle [rechtfertigt schon dieser gegenwärtige Krieg seinen Namen]. Il semble donc qu'elle soit, du point de vue de l'histoire universelle, une transition qui conduit d'une époque européenne révolue vers une époque planétaire à venir. (...) Ce que nous appelons histoire universelle n'est rien d'autre que la transformation de la terre [das Werden der Erde] en un espace historique fermé, un "monde" " "Globus", F. R., Confluences. Politique, bistoire, judaïsme, p. 37, 39 ; Zweistromland. Kleinere Scbriften zu Glauben und Denken, p. 313-314).

2. F. Rosenzweig, Confluences. Politique, bistoire, judaïme, p. 79 ; Zweistromland. Kleinere Schriften zu Glauben und Denken, p. 348. Dans la lettre du 17 février 1917, Rosenzweig révèle qu'il a cité la traduction de Luther "der recbte Kriegsmann» (Moïse 2, 15:3), tandis que "les Juifs nomment, dans leur prière matinale, Dieu Meister der Kriege, Schöpfer des Neuen» (F. R., 1. Briefe und Tagebücher, t. 1, p. 350). 
perduré, qu'il existe encore des régions séparées qui n'appartiennent pas au monde (des régions trop éloignées, qui sont dans le monde et qui, en même temps, n'en font pas partie) et que «l'humanité n'est pas encore rassemblée en une seule demeure [nicht im einen Hause] "'. Peut-être faut-il laisser de côté cette dernière phrase paradoxale sur l'Europe qui ne serait pas encore devenue l'esprit du monde (die Seele der Welt), de même que les réflexions de Rosenzweig sur la Turquie, l'Islam et l'Extrême-Orient, etc., qui apparaissent de temps en temps dans les textes politiques de Rosenzweig. La vision qu'a Rosenzweig du monde et du globe (et non pas seulement de l'Europe) - ainsi formulée, cette vision dépasse de loin certaines analogies et comparaisons séduisantes avec le concept schmittien de Nomos ou avec la distinction schmittienne de la terre et de la mer - demeure inachevée car la guerre n'est tout simplement pas terminée, car elle n'a pas comblé les attentes placées en elle. En d'autres termes, Rosenzweig reste insatisfait de son texte car il reste insatisfait de l'issue de la guerre, de la manière dont elle s'arrête et interrompt sa propre écriture ${ }^{2}$.

Il ne s'agit pourtant pas seulement d'une insatisfaction quant à l'issue de la guerre, courante dans les années d'après guerre. La déception liée à la fin de la guerre, comme conséquence d'un épuisement, d'une mobilisation et d'un patriotisme pluriannuels, pourrait probablement être partagée par Schmitt, Meinecke, Naumann. Tous écrivent à propos de la guerre.

En quoi, chez Rosenzweig, les « usages de la guerre» sont-ils donc différents? Pourquoi son travail sur l'histoire de la raison de la guerre se transforme-t-il soudain, pour quelques mois seulement, en un travail sur la guerre actuelle ? Pourquoi l'écriture sur la Kriegsgrund et le Kriegsziel devient-elle une écriture qui est une attente de l'issue de la guerre (Kriegsausgang)?

Au cours de l'année 1917, au moment même où il l'entame à nouveau (ou bien y revient), Rosenzweig évoque le début de ce projet : cette évocation est particulièrement importante pour nous. Le souvenir des réflexions initiales à propos de ce projet implique un dilemme quant à la question de savoir comment et pourquoi le travail sur ce grand ou petit livre sur la guerre sera par deux fois interrompu et complètement mis au second plan.

1. F. Rosenzweig, Confluences. Politique, bistoire, judaïsme, p. 102 ; Zweistromland. Kleinere Schriften zu Glauben und Denken, p. 368.

2. Ce «drame » entre la guerre et l'écriture se manifeste dans de nombreuses déclarations incontrôlées de Rosenzweig. Tout au long de 1917, il est extrêmement ému et heureux de l'avancée de son texte. La veille de l'écriture de "Thalatta ", il témoigne à quel point le travail préparatif de ce texte est significatif pour lui [Arbeit sebr wichtig], pour finalement dire quatre jours plus tard à ses parents que ce texte ne lui plaît plus du tout [dass mir Thalatta garnicht mehr gefällt]. Au fil du temps, F. R. reproche à ce texte sa forme, puis le fait qu'il ne soit pas scientifiquement «objectif», etc. (F. Rosenzweig, 1. Briefe und Tagebücher, t. 1, p. 497, 502, 504). L'argument de Hilary Putnam, selon lequel Rosenzweig n'a pas voulu publier de son vivant son livre Das Büchlein vom guten und kranken Menschenverstand, parce que celui-ci était anti-philosophique, peut partiellement s'appliquer à « Globus» (H. Putnam, Jewish Philosophy as a Guide to Life, Bloomington, Indiana University Press, 2008, p. 19). 
Est-il donc possible de supposer que l'étude des textes juifs bloquera à deux reprises la construction géopolitique de Rosenzweig? Une première fois, entre les années de doctorat et la guerre, quand il s'introduit lui-même à ces textes, et une seconde fois, à la fin de la guerre, et même à la fin de 1917, quand il demeure insatisfait de ce qu'il a écrit sur la guerre et laisse à nouveau de côté les textes sur l'histoire au profit des livres juifs ${ }^{1}$. Ces deux interruptions, Rosenzweig les marque de la même manière à cinq ou six ans d'écart, en expliquant ce qu'est la théorie juive de la guerre et pourquoi la guerre qui a commencé n'est absolument pas son problème ni son affaire.

Le 9 octobre 1914, il écrit, dans son journal :

«Dans la différence entre le droit à la guerre dans la guerre de religion et dans la guerre profane réside toute l'essence du judaïsme. La chrétienté ne connait que la guerre de religion. Le judaïsme les connait toutes deux également, et si la seconde [la guerre de religion] survient, il ne lui accorde aucun primat sur l'éthique de l'époque. Cela signifie également que la guerre de religion sert à seulement à la fondation de l'existence, tandis que le reste du monde reste "très éloigné de toi". »"

Dans L'Étoile de la Rédemption, qu'il écrit immédiatement après la fin de la guerre, Rosenzweig change quelques mots :

"Aux parties les plus significatives de notre ancienne loi appartient la distinction [Unterscheidung] entre la guerre habituelle [gewöbnlichen Kriegs] contre un peuple "très lointain" [gegen ein "sehr fernes" Volk], menée selon les règles universelles du droit guerrier, guerre qui représente une manifestation normale de la vie pour les formes d'État de même nature, et la guerre sainte [Glaubenskeriegs] contre les "sept peuples" de Canaan, qui permet au peuple de Dieu de conquérir l'espace vital dont il a besoin. (...) Les peuples de l'ère chrétienne [der christlichen Weltzeit] sont incapables de maintenir cette distinction. Conformément à l'esprit du christianisme, qui ne tolère pas de limites [Grenzen], il n'existe pas pour eux de peuples "très lointains". (...) Ils ne sont pas même en mesure de savoir dans quelle mesure une guerre est une guerre sainte ou simplement guerre profane [bloss weltlicher Krieg]. Mais, en tous cas, ils savent qu'en quelque manière [irgendwie] la volonté de Dieu s'accomplit dans les destinées guerrières de leurs États [in den kriegerischen Geschicken ibres Staates

1. Cf. lettre du 26 décembre 1917, 1. Briefe und Tagebücher, t. 1, p. 501-503.

2. In der Scheidung des Kriegsrechts in Religions- und Profankrieg (5. Mose 20, 15-16) steckt das ganze Wesen des Judentums. Das Christentum kennt nur den Religionskrieg. Das Judentum kennt beide nebeneinander und erbebt sich in der Ethik des zweiten nicht über die Ethik des Zeitalters. Auch bezeich-

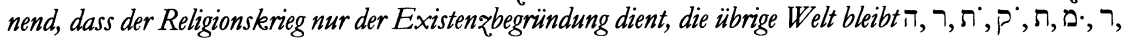
D, $\mathrm{n}$.

"So tue allen Städten "sehr entfernt von dir" [heraym harchokot mimecha me'od]. Moise, V, 20:15. » F. Rosenzweig, 1. Briefe und Tagebücher, t. 1, p. 175-176. "Ainsi procéderas-tu pour toutes les villes situées très loin de chez toi, qui ne font point partie des villes de ces nations" (Rabbinat français). Au sujet de cette distinction, plus actuelle que jamais depuis l'existence d'Israël, voir les textes de J. D. Bleich, "Preemptive war in Jewish law ", Tradition, 21, n. 1, 1983, p. 3-41 ; G. B. Levey, " Judaism and the obligation to die for the State ", AJs Review, 12, n. 2, 1987, p. 175-203; M. Walzer, "The idea of Holy War in Ancient Israel ", Journal of Religious Ethics, 20, n. 2, 1992, p. 215-228; M. Walzer, "War and peace in the Jewish tradition ", in T. Nardin (ed.), The Ethics of War and Peace, Princeton, Princeton University Press, 1996, p. $95-113$. 
verwirklicht]. En quelque manière... La manière demeure mystérieuse [Irgendwie - das Wie bleibt rätselhaft] (...) la guerre seule décide [entscheidet], la guerre qui passe sans égard sur la conscience de l'individu. $\nu^{1}$

«En quelque manière... La manière demeure mystérieuse... » Peut-être faudrait-il traduire : "La manière demeure énigmatique. »

Comment (wie) la volonté de Dieu se réalise-t-elle? Comment Dieu gouverne-t-il les États (les États chrétiens)? Comment (wie) Dieu décide-t-il de la guerre? Comment (wie)?

L'énigme de ces questions explique à elle seule tout l'effort de Rosenzweig et son projet des années précédentes. Il n'y a pas une seule phrase dans ce célèbre extrait du livre de Rosenzweig qui n'ait pas été écrite d'une manière ou d'une autre dans ses journaux, lettres ou textes de la guerre. De plus, cette énigme qui tourmente Rosenzweig et qui est le secret de son " engagement guerrier" (énigme qui entretient un «lien » incompréhensible avec les peuples et États chrétiens - qui risquent de disparaître dans la guerre - et avec Dieu, qui décide et les gouverne), Rosenzweig la place immédiatement au second plan. En effet, les deux paragraphes suivants sont une indication extrêmement claire adressée au peuple juif, mais aussi au Juif, à Rosenzweig, à nous ou à qui que ce soit qui souhaite à l'avenir s'intéresser à la raison de la guerre. De même que le projet de Rosenzweig est pénétré de textes juifs d'avant et d'après guerre, de même, dans le texte de L'Étoile de la Rédemption, cette énigme (das Rätsel) se trouve « entre» le savoir de la différence des guerres ("l'essence de la judaïté », selon les mots de Rosenzweig) et ce message de Rosenzweig au peuple juif :

"Mais comme il [le peuple juif] possède malgré tout le concept de guerre sainte, il ne peut prendre ces guerres (guerres purement politiques) au sérieux... [ernst nebmen] (...). De fait, à l'intérieur du monde chrétien, le juif est proprement la seule personne incapable de prendre la guerre au sérieux [nicbt ernst nehmen kann], et en ce sens il est le seul "pacifiste" authentique [der einzige echte "Pazifist" $]^{2}$. [...] le peuple juif se tient en dehors du monde [steht es ausserhalb der Welt] (...) en vivant la paix éternelle, il se tient en dehors d'une temporalité guerrière [stebt es ausserbalb ener kriegerischen Zeitlichkeit]. ."

Il semble que cette surprenante résistance envers la temporalité du monde et de la guerre est susceptible de mieux expliquer l'intensité de l'effort de Rosenzweig au cours des années précédentes. Le livre sur la guerre qui n'a pas été écrit (Ein Buch über den Krieg) est seulement l'épilogue

1. F. Rosenzweig, L'Étoile de la Rédemption, trad. J.-L. Schlegel et A. Derczanski, Paris, Le Seuil, 2003, p. 461 ; Der Stern der Erlösung, Frankfurt am Main, Suhrkamp, 1996, p. 367.

2. Les guillemets de "Parifist» existent également dans la première édition du livre $D e r$ Stern der Erlösung, Frankfurt am Main, J. Kauffmann Verlag, 1921, p. 416. Je prépare actuellement un texte sur la nature de cette reconstruction de Rosenzweig du " "pacifiste" authentique » [der einzige ecbte "Pazifist "), et sur son interprétation du pacifisme dans ses journaux et sa correspondance. Cf. F. Rosenzweig, 1. Briefe und Tagebücher, t. 1, p. 183, 204, 210-214, 326-328.

3. F. Rosenzweig, L'Étoile de la Rédemption, p. 462 ; Der Stern der Erlösung, p. 368. 
d'un «surinvestissement messianique " extrêmement complexe de Rosenzweig. Quelques conditions sont nécessaires à la résolution de l'énigme, que Rosenzweig a intensivement préparée dans l'attente de la guerre ou du jugement dernier (göttliche Gericht) ${ }^{1}$ : il est nécessaire, insiste Rosenzweig, de penser « cette " guerre comme si [als ob] c'était la dernière ${ }^{2}$; de fuir le chauvinisme naiff de déployer une pensée catastrophique [katastrophal denken], et non seulement "différentielle " [differential $\beta$; la différence et l'unité entre la guerre, "le père de toutes choses ", et la paix, "la mère de toutes choses ", est comblée dans le destin du monde; la raison secrète [der geheime Grund] $]^{4}$ de la guerre (mondiale) et de l'histoire réside dans le fait que les peuples sont à la recherche de leur esprit, d'un monde sans frontières et d'un monde sans peuples (éloignés); la raison de la guerre [Kriegsgrund] se superpose toujours avec sa finalité [Kriegsziel] ${ }^{5}$; le Messie arrive seulement au terme guerrier de l'histoire du monde [nur im kriegerischen Ablauf der Weltgeschichte]; "aujourd'hui » arrive le Messie ["Heute "kommt Messias].

Pourtant, "aujourd'hui " "n'est pas encore le véritable “aujourd'hui" " [es ist noch nicht das rechte "Heute »]'

Petar BOJANIC. (Traduit par Igor Krtolica.)

1. "Der Krieg ist ein "göttliches Gericht", aber kein einfaches Strafgericht, sondern "Krisis", Scheidung, Böcke und Schafe" (lettre du 17 février 1917 ; F. R., 1. Briefe und Tagebücher, t. 1, p. 350).

2. "Paralipomena », F. R., 3. Zweistromland. Kleinere Schriften qu Glauben und Denken, p. 90. Cf. F. P. Ciglia affirme que ces fragments de Rosenzweig, retrouvés il y a trente ans, ont été écrits en 1917, et non en 1916 (F. R., Globus. Per una teoria storico-universale dello spazio, p. 126).

3. "Paralipomena ", F. R., 3. Zweistromland. Kleinere Scbriften zu Glauben und Denken, p. 72.

4. F. Rosenzweig, lettre du 3 juillet 1918, 1. Briefe und Tagebücher, t. 1, p. 586. Dans la construction de ces surinvestissements «messianiques » de Rosenzweig, j’ai utilisé le livre de Daniela Toti, Franz Rosenzweig : possibilità di una fondazione della nuova filosofia nella storia, Roma, Gregoriana, 2000, p. 202-209.

5. F. Rosenzweig, " Globus », Confluences. Politique, histoire, judaïsme, p. 100 ; Zweistromland. Kleinere Schriften zu Glauben und Denken, p. 366.

6. «Paralipomena », F. R., 3. Zweistromland. Kleinere Schriften zu Glauben und Denken, p. 91. 